\title{
BMJ Open Early outcomes of a rural-oriented physician education programme against rural physician shortages in Guangxi province: a prospective cohort study
}

\author{
Ying Shen (D) , ${ }^{1}$ Xing Huang, ${ }^{2}$ Hong Li, ${ }^{1}$ Enran Chen, ${ }^{1}$ Yan Kong, ${ }^{1}$ Jia Yu, ${ }^{1}$ \\ Xiaoyun Liu (D) , ${ }^{3}$ Siam Al Mobarak, ${ }^{4}$ Yanli Zuo ${ }^{1}$
}

To cite: Shen $Y$, Huang $X$, Li H, et al. Early outcomes of a rural-oriented physician education programme against rural physician shortages in Guangxi province: a prospective cohort study. BMJ Open 2021;11:e049274. doi:10.1136/ bmjopen-2021-049274

- Prepublication history for this paper is available online. To view these files, please visit the journal online (http://dx.doi org/10.1136/bmjopen-2021 049274).

Received 20 January 2021 Accepted 21 August 2021

Check for updates

C Author(s) (or their employer(s)) 2021. Re-use permitted under CC BY-NC. No commercial re-use. See rights and permissions. Published by BMJ.

${ }^{1}$ School of General Practice, Guangxi Medical University, Nanning, Guangxi, China ${ }^{2}$ Division of Teaching Affairs, Guangxi Medical University, Nanning, Guangxi, China ${ }^{3}$ China Center for Health Development Studies, Peking University, Beijing, China

${ }^{4}$ The Department of Cardiology, Guangxi Medical University First Affiliated Hospital, Nanning, Guangxi, China

Correspondence to

Professor Yanli Zuo;

zuoyanli@gxmu.edu.cn

\section{ABSTRACT}

Objectives This study aimed to investigate early outcomes of one of the first medical undergraduate education programmes with a goal of mitigating severe rural physician shortages in China, which was developed by Guangxi Medical University (GXMU) and was called the Rural-oriented Free Tuition Medical Education (RTME)GXMU programme.

Design A prospective cohort study comprising a baseline investigation and follow-up research was conducted to dynamically observe the evolution of the RTME-GXMU programme that began since 2010.

Participants 380 RTME-GXMU graduates and 383 non-RTME-GXMU graduates from GXMU who completed trainings between 2015 and 2018 were recruited in the baseline investigation. Among them, 285 RTME-GXMU and 283 non-RTME-GXMU graduates responded to the followup research.

Main outcome measures Graduate practice location, registered specialty, passing rate of the National Medical Licensing Examination (NMLE), specialty of residency programme and contract compliance for the RTME-GXMU graduates.

Results By the end of $2018,100 \%$ of the 2015 RTMEGXMU graduates enrolled in this study practised in rural township health centres and registered themselves as general practitioners (GPS). All the RTME-GXMU graduates had completed or were attending residency programmes of general practice (GP). The above data stood in stark contrast to that of the non-RTME-GXMU graduates among whom as few as $1.06 \%$ worked in rural areas, $2.13 \%$ registered as GPs and less than 3\% chose GP residency programmes. No significant differences were detected on passing rates of the NMLE between the two groups. Only one RTME-GXMU graduate broke the contract and dropped off the programme.

Conclusions The RTME-GXMU programme has achieved encouraging early outcomes. Reduced entry score and proper usage of urban primary care institutions are two key approaches contributing to these positive early results.

\section{INTRODUCTION}

The physician shortage in rural areas remains a daunting global challenge facing both developed and less developed countries. The
Strengths and limitations of this study

- It is the first comprehensive and longitudinal study in China to evaluate early outcomes of a medical undergraduate education programme dedicated to mitigating rural physician deficiency.

- Experiences regarding admission and clinical curriculum development of the Rural-oriented Free Tuition Medical Education-Guangxi Medical University (RTME-GXMU) programme offer insights into how to effectively organise a medical education programme with the purpose of mitigating rural physician shortages in low and middle-income countries.

- Engagement of local health administrations increases accuracy of data on the RTME-GXMU graduates.

- It is a prospective cohort study that provides a direct and dynamic observation on the RTME-GXMU programme.

- This study did not measure long-term retention of the RTME-GXMU graduates after the obliged service because it will be due in 2021 .

physician workforce deficiency has inevitably diminished access to healthcare in rural areas and has compromised health outcomes of rural residents. ${ }^{12}$ In China, rural physician shortages feature a deficit both in quantity and in quality. ${ }^{3}$ Around $40.42 \%$ of population in China lived in rural areas by the end of $2018,{ }^{4}$ while only $33.71 \%$ of health professionals were practising there. ${ }^{4}$ Rural areas possessed 1.82 physicians plus assistant physicians (AP) per 1000 population $^{4}$ in 2018 , less than half the rate of urban regions which reached up to 4.01 in the same year. ${ }^{4}$ Among physicians and APs practising in rural township health centres, as few as $17.3 \%$ were certificated a Bachelor of Medicine degree in 2017 which stood in stark contrast to $40.1 \%$ in urban primary healthcare centres and even $50.1 \%$ in urban hospitals. ${ }^{5}$ Consequently, when compared with their urban counterparts, rural inhabitants lagged behind in many 
crucial health parameters including newborn mortality rate (4.7 vs 2.2), infant mortality rate (7.3 vs 3.6), underfive mortality rate (10.2 vs 4.4 ) and maternal mortality rate (19.9 vs 15.5). ${ }^{4}$ Moreover, the scale of Chinese elderly people over 65 years exceeded $9 \%$ of the total population in 2011 and had been growing steadily since then, ${ }^{6}$ which marked the existence of an ageing society and heralded increasing demands on primary healthcare as well as longterm care. Against the above background, the question of how to effectively address physician shortages in rural China presents much more urgency than ever before.

Specific medical education programmes targeting the rural physician deficiency in the USA, ${ }^{7}$ Canada, ${ }^{8}$ Japan, ${ }^{9}$ Australia ${ }^{10}$ and Thailand $^{11}$ have demonstrated a range of favourable impacts on improving supply and performance of physicians as well as other health professionals. In light of these international productive cases, a national rural-oriented physician education policy by the name of 'Rural-oriented Free Tuition Medical Education (RTME)' has been issued in China in 2010 and has been proceeding for nearly a decade. The RTME exclusively recruited students with rural backgrounds and subsidised them to finish a 5-year medical undergraduate education. In return, these RTME students must attend a 3-year residency programme in general practice (GP) and compulsorily serve in rural township health centres for a period of 3 years. At the very beginning, the RTME targeted 23 provinces in middle and western China that suffered severe rural physician shortages and commissioned 51 medical universities and colleges nationwide to develop detailed training programmes. ${ }^{12}$ Since 2018, the first batch of general practitioners (GPs) fostered by the RTME has been actively serving local rural inhabitants.

Guangxi province was among the 23 beneficiary provinces sponsored by the RTME. It has been suffering a persistent rural physician shortage with the rate of physician and AP per 1000 population in rural areas less than the national average over the last 8 years. ${ }^{4}$ In 2010 , granted by the central government and the Guangxi provincial government, the General Practice School of Guangxi Medical University (GXMU) developed one of the nation's first rural-oriented physician education programmes under the frame of the RTME, the RTMEGXMU programme. Since then, the RTME-GXMU programme has been devoted to its original mission to cultivate GPs who intend to practise in local rural areas and are competent for rural healthcare settings.

This study aimed to evaluate early outcomes of the RTME-GXMU programme, which principally concentrated on examining the academic performance and contract fulfilment of the RTME-GXMU graduates. Graduates between 2015 and 2018 from both the RTMEGXMU and the non-RTME-GXMU were enrolled as study participants. In order to properly assess early outcomes, a variety of parameters comprising demography, rural practice location, registered specialty, passing rate of the National Medical Licensing Examination (NMLE) and specialty of residency programme were investigated and compared in a prospective cohort study that conducted a baseline investigation and follow-up research. To our best knowledge, this study is the first attempt in China to evaluate the effectiveness of a medical undergraduate education programme aimed to mitigate rural physician deficiencies, in particular to carry out such an evaluation after a whole cultivation cycle that stretches across 5 years of medical undergraduate education plus 3 years of residency programme in GP.

\section{RTME-GXMU programme components}

Admission policy

From the very beginning, the RTME-GXMU programme has established a rural-oriented admission policy that prioritises enrolment of students born and raised in local rural areas, reduction on entry score requirement and commitment to mandatory services in rural areas. In 2010, as assigned by the Guangxi Provincial Health Commission, the RTME-GXMU programme admitted a total of 100 students from rural areas governed by seven cities within the Guangxi province: Nanning, Liuzhou, Beihai, Qingzhou, Fangchenggang, Yulin and Guigang. The average level of entry scores on the National College Entrance Examination (NCEE) for these first 100 students was significantly lower than for non-RTME-GXMU students of clinical medicine in GXMU. Decreasing the threshold of admission by implementing lower acceptance scores was done to maximise the recruitment of as many potential candidates as possible. But it also raised a wide concern that inferior recruitment criteria might compromise outcomes of the medical education programme. Nonetheless, the RTME-GXMU students would receive higher standard medical education that combined undergraduate medical education and residency programme than did the current rural physicians despite of lower admission scores. Each student admitted to the RTME-GXMU programme was required to sign a contract with local rural health administrations. The contract defined that the RTME-GXMU students were given free tuition for medical undergraduate education as well as stipends for their living expense in exchange for their attendance at residency programmes in GP and fulfilment of obliged rural services. The admission size remained as 100 students per year from 2010 to 2016, and expanded to 160 per year since 2017 and to 260 since 2019. The number of source cities on admission has increased to 8 since 2011 with Liuzhou removed from the list and another two cities, Chongzuo and Wuzhou, added in.

\section{Curriculum}

A distinct ' $3.5+1.5$ ' curriculum underscoring rural health experience and primary healthcare training has been developed and identified as an unique feature of the RTME-GXMU programme. Under the curriculum, the RTME-GXMU students spent the first three and a half years out of a 5-year medical undergraduate education learning all the courses required of other GXMU medical 
students in 4years, but, in addition, received courses related to rural health and GP. These extra courses consisted of lectures, flipped classes, e-learning at the campus of GXMU, as well as clinical clerkship in local urban primary healthcare centres which extended across the first two and a half years. During the clinical clerkship that usually occupied the last one and a half years, the RTME-GXMU students rotated in rural county hospitals and urban primary healthcare centres respectively for 8 consecutive weeks. At the summer break of every year, a social event named 'Experiencing the Rural' aimed at increasing rural health experiences was held and brought the first and second year RTME-GXMU students to rural township health centres or village clinics. Students lived there for around a week with rural physicians and shadowed them working in rural settings. When compared with a ' $4+1$ ' curriculum which is the mainstream medical education pattern in China and combines a 4-year theorybased learning and a 1-year clerkship that usually takes place in highly specialised urban hospitals, the ' $3.5+1.5$ ' curriculum allows much more time and opportunities for students to be trained in the fields of rural health and primary healthcare. The clinical clerkship is prolonged by half a year to allow for rotations in rural health settings and primary healthcare facilities. This is widely acknowledged as a bold and creative innovation in Chinese medical education history.

\section{METHODS}

\section{Participants and study design}

With the permission of the GXMU Ethics Commission, two cohorts were established in 2015 for a longitudinal survey that intended to evaluate early and long-term outcomes of the RTME-GXMU programme. The RTMEGXMU cohort enrolled all graduates from the programme every year since 2015 when the first participants graduated. The non-RTME-GXMU cohort accumulated other clinical graduates of GXMU during the same period on the ratio of 1 vs 1 . If graduates failed to provide either written informed consents or graduation certificates, they were excluded from the two cohorts. A prospective cohort study consisting of a baseline investigation and follow-up research was conducted to appraise the RTMEGXMU programme. The baseline investigation included all graduates within the above two cohorts who graduated from 2015 to 2018, and examined yearly baseline information including demography and acceptance scores. The ensuing follow-up research, from 2016 to 2018, investigated the specialty of residency programme for graduates from the RTME-GXMU and the non-RTME-GXMU cohorts every year and compared possible differences between the two. From 2016 to 2018, the follow-up study collected the passing rates of the NMLE for all the clinical graduates of GXMU who attended the NMLE at the first attempt in the very year. At the end of 2018, when the first batch of RTME-GXMU graduates who finished the 5 years of medical undergraduate education together with the 3 years of residency training came back to rural township health centres and started fulfilling the obligatory services, the follow-up research detected the rural practice location and the registered specialty of the graduates recruited in 2015 in both cohorts. Meanwhile, the number of the RTME-GXMU graduates who broke the contracts from 2015 to 2018 was collected.

\section{Data collection}

Demography and entry scores over both cohorts were acquired every year from the Enrollment and Employment Division of GXMU, and passing rates of the NMLE from 2016 to 2018 of all the clinical graduates from the Teaching Affairs Division of GXMU. Data regarding specialty of residency programme, rural practice location, as well as registered specialty on the RTME-GXMU cohort were obtained from the Health Commission of Guangxi Province. Data concerning specialty of residency programme on the non-RTME-GXMU cohort were collected every year through a questionnaire developed by the authors, and data regarding rural practice location and registered specialty were collected through another self-made questionnaire in 2018. The number of RTMEGXMU graduates who broke their contracts was obtained from the Health Commission of Guangxi Province.

\section{Data analysis}

Quantitative data were recorded by EpiData and analysed by SPSS Statistical Software V.22. Pearson's $\chi^{2}$ was employed to compare the passing rates of the NMLE, gender, rural background and minor group between the RTME-GXMU graduates and the non-RTME-GXMU graduates. Fisher's exact test was used to analyse the percentages of graduates practising in rural areas, registering as GP and choosing residency programme in GP. The t-test was adopted to compare age as well as acceptance scores between both types of graduates. A p value of 0.05 or less was regarded significant in all comparisons.

\section{Patient and public involvement}

No patients were involved in this study.

\section{RESULTS}

\section{Samples of the baseline investigation and the follow-up} research

A total of 380 graduates from the RTME-GXMU cohort and 383 from the non-RTME-GXMU cohort who graduated from 2015 to 2018 were included in the baseline investigation. Among them, 191 graduates were enrolled in 2015; 186 in 2016; 195 in 2017; and 191 in 2018. A total of 285 RTME-GXMU and 283 non-RTME-GXMU graduates participated in the follow-up research. Both of the two categories of graduates showed high follow-up rates from 2016 to 2018. The number of non-RTME-GXMU graduates who responded to the questionnaire regarding specialty of residency programme totalled 94 in 2016; 92 in 2017; and 97 in 2018, and those who responded to 
Table 1 Sample distribution of participants in the baseline and follow-up studies

\begin{tabular}{|c|c|c|c|c|c|c|c|c|c|c|}
\hline & \multicolumn{5}{|c|}{ RTME-GXMU graduates } & \multicolumn{5}{|c|}{ Non-RTME-GXMU graduates } \\
\hline & 2015 & 2016 & 2017 & 2018 & 2018 & 2015 & 2016 & 2017 & 2018 & 2018 \\
\hline Baseline recruitment & 96 & 94 & 96 & 94 & - & 95 & 92 & 99 & 97 & - \\
\hline Rate of follow-up (\%) & & 98.96 & 100 & 100 & 100 & & 98.95 & 100 & 97.98 & 98.95 \\
\hline Lost follow-up & & 1 & 0 & 0 & 0 & & 1 & 0 & 2 & 1 \\
\hline
\end{tabular}

${ }^{*}$ The number of graduates who graduated in 2015.

†The number of graduates who graduated in 2016.

$\ddagger$ The number of graduates who graduated in 2017.

GXMU, Guangxi Medical University; RTME, Rural-oriented Free Tuition Medical Education.

rural practice site as well as registered specialty totalled 94 in 2018, with respondence rates as $98.95 \%, 100 \%$, $97.98 \%$ and $98.95 \%$. For a detailed sample distribution, see table 1 .

\section{Demography and acceptance score on admission}

From 2015 to 2018, a significantly higher proportion of the RTME-GXMU graduates $(80.53 \%)$ indicated rural backgrounds than did the non-RTME-GXMU graduates (60.31\%) when both were admitted to the GXMU. A lower average level of acceptance scores on the NCEE was revealed in the RTME-GXMU graduates $(522.26 \pm 22.8)$ than in the non-RTME-GXMU graduates $(587.34 \pm 31.57)$. No significant differences were detected between the above two groups with respect to age, gender or minor ethnic group. For detailed data, see table 2.

Passing rate of the NMLE, specialty of residency programme, practice location and registered specialty

The passing rate of the NMLE in the RTME-GXMU graduates was $92.55 \%$ in $2016,91.58 \%$ in 2017 and $89.36 \%$ in 2018 separately, indicating no significant differences when compared with the non-RTME-GXMU graduates $(91.89 \%$ in 2016, 90.88\% in 2017 and $87.78 \%$ in 2018, respectively). All these passing rates of the NMLE revealed in the two kinds of graduates notably exceeded the national average levels for 3 years straight (see table 3). From 2016 to $2018,100 \%$ of the RTME-GXMU graduates responding to the follow-up research chose residency programmes in GP. This rate overwhelmingly surpassed that of the nonRTME-GXMU graduates who made the identical options during the same period (see table 4). By the end of 2018, $100 \%$ of the 2015 RTME-GXMU graduates had practised in rural township health centres after they completed the 3-year residency programmes in GP, while only one $(1.06 \%)$ non-RTME-GXMU graduate had reported practising in rural site, which drew a stark comparison. The subsequent comparison regarding registered specialty repeated the above significant gap when $95(100 \%)$ RTME-GXMU graduates registered themselves as GPs and only two $(2.13 \%)$ non-RTME-GXMU graduates did so (see table 5).

\section{Contract fulfilment of the RTME-GXMU graduates}

At the end of 2018, only one out of 380 RTME-GXMU graduates broke the contract according to the Health Commission of Guangxi Province. Thus, $99.73 \%$ of the RTME-GXMU graduates included in this study had kept the contracts, an extremely high rate of contract compliance.

\section{DISCUSSION}

As indicated in the results, the RTME-GXMU programme has achieved strongly positive early outcomes. All 95 RTME-GXMU graduates followed up in this study who graduated in 2015 have completed 5 years of medical undergraduate education plus 3 years of residency training in GP and have started fulfilling their service obligations in rural primary healthcare facilities since 2018. Each of the RTME-GXMU graduates currently practising

Table 2 Demography on admission between graduates of the RTME-GXMU and non-RTME-GXMU

\begin{tabular}{llll}
\hline Demography & RTME-GXMU graduate, $\mathbf{n = 3 8 0}$ & Non-RTME-GXMU graduate, $\mathbf{n = 3 8 3}$ & P value \\
\hline Age $($ years), mean $(\overline{\mathrm{x}} \pm \mathrm{s})$ & $18.20 \pm 0.66$ & $18.30 \pm 1.00$ & 0.66 \\
Gender, female, $\mathrm{n}(\%)$ & $179(47.11)$ & $199(51.96)$ & 0.09 \\
Rural ${ }^{*}$ background, $\mathrm{n}(\%)$ & $306(80.53)$ & $231(60.31)$ & $<0.0001$ \\
\hline Minor ethnic, $\mathrm{n}(\%)$ & $151 / 380(39.74)$ & $136 / 383(35.51)$ & 0.11 \\
Acceptance score, $\operatorname{mean}(\overline{\mathrm{x}} \pm \mathrm{s})$ & $522.26 \pm 22.8$ & $587.34 \pm 31.57$ & $<0.0001$
\end{tabular}

*Graduates of RTME-GXMU or non-RTME-GXMU who had lived in rural areas during 1-15 years when admitted to the GXMU. GXMU, Guangxi Medical University; RTME, Rural-oriented Free Tuition Medical Education. 
Table 3 Passing rates of the NMLE over the RTME-GXMU and non-RTME-GXMU graduates

\begin{tabular}{|c|c|c|c|c|c|c|c|c|}
\hline \multirow[b]{2}{*}{ Year } & \multicolumn{3}{|c|}{ RTME-GXMU graduates* } & \multicolumn{3}{|c|}{ Non-RTME-GXMU graduates $\dagger$} & \multirow[b]{2}{*}{$P$ value } & \multirow[b]{2}{*}{$\begin{array}{l}\text { National average } \\
\text { level of passing } \\
\text { rate }(\%)\end{array}$} \\
\hline & $\begin{array}{l}\text { Graduates } \\
\text { attending } \\
\text { the NMLE* }\end{array}$ & Passers & $\begin{array}{l}\text { Passing rate } \\
(\%)\end{array}$ & $\begin{array}{l}\text { Graduates } \\
\text { attending the } \\
\text { NMLE† }\end{array}$ & Passers & $\begin{array}{l}\text { Passing rate } \\
(\%)\end{array}$ & & \\
\hline 2016 & 94 & 87 & 92.55 & 666 & 612 & 91.89 & 0.48 & 73.75 \\
\hline 2017 & 95 & 87 & 91.58 & 647 & 588 & 90.88 & 0.48 & 67.64 \\
\hline 2018 & 94 & 84 & 89.36 & 610 & 610 & 87.78 & 0.44 & 70.38 \\
\hline
\end{tabular}

*The number of graduates of the RTME-GXMU who were at the first attempt of the NMLE in 2016, 2017 and 2018 , respectively.

†The number of graduates of non-RTME-GXMU who were at the first attempt of the NMLE in 2016, 2017 and 2018, respectively.

GXMU, Guangxi Medical University; NMLE, National Medical Licensing Examination; RTME, Rural-oriented Free Tuition Medical Education.

in rural areas identified GP as their practice specialty. All 285 RTME-GXMU graduates who attended the follow-up research chose GP as their residency specialty. These data demonstrated a stark contrast between the RTME-GXMU and the non-RTME-GXMU graduates. As few as $1.06 \%$ of the non-RTME-GXMU graduates who responded to this study practised in rural areas, only $2.13 \%$ registered themselves as GPs and less than 3\% once were trained in GP residency programmes. Both groups of graduates showed similar passing rates on the NMLE, without significant differences detected, even though the entry scores for the RTME-GXMU graduates were much lower than for the non-RTME-GXMU trainees. In addition to qualified academic abilities, the RTME-GXMU graduates displayed far stronger interests and intentions towards serving in rural township health centres than did the non-RTMEGXMU ones.

These promising early outcomes strongly suggested the positive impact of the RTME-GXMU programme in fostering competent rural physicians and relieving rural physician deficiencies in the local. Extremely high proportions of graduates practising in rural sites and successfully passing NMLE exhibited that the RTME-GXMU programme has achieved positive early outcomes consistent with other medical education programmes with similar commitment requirements in the USA, ${ }^{13}{ }^{14}$ Australia, ${ }^{15}{ }^{16} \mathrm{Japan}^{17}$ and Thailand. ${ }^{18}$ The above-mentioned foreign medical education programmes have obtained success at different extents in promoting recruitment and retention of the rural physician workforce in their own countries.
The RTME-GXMU programme adds new evidence to what a positive role a medical education programme could play in decreasing rural physician shortages within multiple disparate societies that stand at different stages of socioeconomic development, and with different education and health systems. As a contract-based medical education programme, the RTME-GXMU programme showed extraordinarily high contract compliance among its graduates. Only one graduate broke the contract signed by the local health administration and left the RTME-GXMU programme soon after graduation without admittance to the required residency programme. The great majority of the RTME-GXMU graduates were on track during the time of this study. Multiple medical education programmes ${ }^{19-21}$ that were contract binding and attached with obliged rural services reported as high a compliance rate of graduates as did the RTME-GXMU programme. The Jichi Medical University programme of Japan ${ }^{19}$ further identified factors associated with obligation compliance and concluded that primary care specialty positively influenced the obligation compliance. Specialty of primary healthcare also was reported to promote recruitment and retention of rural physicians in the $\mathrm{USA}^{13}$ and Australia. ${ }^{22}$ This may, in part, explain why the RTME-GXMU graduates are highly compliant with their contracts. GPs dominated the registered specialty and residency specialty of the RTME-GXMU graduates. GPs typically are more desirable in rural health settings and more recognised in rural healthcare than other specialists, therefore they would stick to rural health settings longer than other specialists would do. However,

Table 4 Attendance of residency programme of general practice between the RTME-GXMU and non-RTME-GXMU graduates

\begin{tabular}{llll}
\hline & RTME-GXMU graduates & Non-RTME-GXMU graduates & P value \\
\hline $2016, \mathrm{n}(\%)$ & $95 / 95(100.00)$ & $2 / 94(2.13)$ & $<0.0001$ \\
$2017, \mathrm{n}(\%)$ & $94 / 94(100.00)$ & $1 / 92(1.09)$ & $<0.0001$ \\
$2018, \mathrm{n}(\%)$ & $96 / 96(100.00)$ & $2 / 97(2.06)$ & $<0.0001$ \\
\hline
\end{tabular}

GXMU, Guangxi Medical University; RTME, Rural-oriented Free Tuition Medical Education. 
Table 5 Practice location, registered specialty between the RTME-GXMU and non-RTME-GXMU graduates

\begin{tabular}{llll} 
& RTME-GXMU graduate & Non-RTME-GXMU graduate & P value \\
\hline Rural practice site, n, \% & $95,100 \%(95 / 95)$ & $1,1.06 \%(1 / 94)$ & $<0.0001$ \\
Registered as family physician, n, \% & $95,100 \%(95 / 95)$ & $2,2.13 \%(2 / 94)$ & $<0.0001$ \\
\hline
\end{tabular}

GXMU, Guangxi Medical University; RTME, Rural-oriented Free Tuition Medical Education.

given the distinctively different features of society and culture in China, further studies are required to recognise specific elements associated with contract compliance of medical graduates in the unique context of China.

It is well established that recruitment of students with rural backgrounds and implementation of long-term rural setting clerkships are two key strategies ${ }^{23} 24$ in medical education programmes to contribute to enhanced recruitment and long-term retention of the rural physician workforce. Besides the above two strategies, the RTME-GXMU programme adopted two additional approaches that were effective and based on local rural health environments and medical education conditions. The first approach was to reduce the minimum entry score requirements of the RTME-GXMU programme. The point of the reduction was to attract as many candidates as possible who were truly interested in rural health and intended to practise in rural areas in the future. The average entry scores of students recruited to the RTME-GXMU were lower than that of other clinical students enrolled to GXMU by around 60 credits from 2010 to 2013 . The significant gap of entry score between the RTME-GXMU and the nonRTME-GXMU students once raised extensive concerns over student performance in the NMLE and even clinical performance in rural primary healthcare settings. The fact that the passing rates of NMLE in the RTME-GXMU graduates were not statistically different from those of the non-RTME-GXMU graduates and far above the average levels of the whole China shattered those previous worries. The extremely high passing rates on the NMLE have put in question to association between the entry score and passing the NMLE. Reduced threshold of entry score enhanced the recruitment of the RTME-GXMU by widening the access to more potential rural students.

The second approach was to make full use of urban primary healthcare facilities. The clerkship that occupied the last one and a half years in the RTME-GXMU programme comprised rotations in urban teaching hospitals, rural hospitals, as well as urban primary healthcare centres. Rotations in rural primary healthcare institutions were not included in the clerkship, which would have deprived the RTME-GXMU students of working in locations resembling where they were supposed to practise for 3years after finishing their GP residency programmes. Incompetent faculty, substandard training conditions and limited educational resources were three main barriers to keep rural primary healthcare facilities out of the list of clerkship sites. Actually, rural primary healthcare facilities are usually applied to trainings of APs but not of physicians in China and none of the Chinese literature has reported the usage of rural township health centres in physician cultivation. Therefore, rural hospitals, together with urban primary healthcare centres, become a common training pattern to get the students' rural health experiences in medical education and residency programmes dedicated to fostering rural GPs. The RTME-GXMU programme has proven that the absence of clerkships in rural primary healthcare institutions does not negatively influence the passing rates of NMLE, GP residency selection, registration as a GP or practice in rural areas. In China, urban community health service centres and rural township health centres are categorised as primary healthcare institutions and are assumed to provide primary healthcare to local residents. They both technically have similar systems, organisation, functions, as well as professional constitution. However, the former generally is equipped with better faculty and teaching resources than is the latter. Urban primary healthcare organisations can be a makeshift, in particular when teaching conditions of rural primary healthcare organisations are too limited to be qualified for physician training. However, remarkable differences between rural and urban primary healthcare do exist, which cannot be ignored. Moreover, a number of previous research ${ }^{25-29}$ indicated that the long-term rural clerkship experience was positively associated with improved long-term retention of physicians in rural areas. With the first graduates beginning practices in rural township health centres, now comes the best chance for the RTME-GXMU programme to construct a clerkship in rural township health settings. The RTME-GXMU graduates currently working in rural township health centres can be potential sources to competent faculty of a clerkship set up in rural primary health settings. Moreover, a steady growth in investments by national and local governments on rural health institutions in the last decade has substantially improved the teaching conditions of rural health settings. How to establish an integrated clerkship curriculum that highlights the rural primary healthcare service will be one of the priorities of the RTME-GXMU in a bid for further improvements.

This study followed the design of a prospective cohort research to evaluate the early outcomes of the RTMEGXMU programme. Compared with most studies assessing the effectiveness of medical education programmes with a specific view to remitting rural physician deficiencies, the prospective cohort study provided a direct and dynamic observation on the RTME-GXMU programme. This study 
is the first attempt in China to assess comprehensively and longitudinally a medical undergraduate education programme aimed at improving severe rural physician deficiencies, and to introduce practical experiences for development and implementation of a rural-oriented medical education programme in low and middleincome countries. There are a number of limitations to this study, including the short time frame of the study and the observational nature. However, the primary limitation is that the initial cohort has not completed their 3-year commitment to rural service. So complete fulfilment of the contracts and retention after the obliged rural service cannot be evaluated during this study.

\section{CONCLUSION}

The RTME-GXMU programme has exhibited encouraging early outcomes that present extremely high rates of graduates practising in rural primary health facilities and passing the NMLE. Reduced entry scores to expand recruitment and proper usage of urban primary healthcare institutions are two key approaches contributing to positive early outcomes. Further studies are required to explore long-term retention of the RTME-GXMU graduates in rural areas after 3 years of obligatory service and potential links between the RTME-GXMU and retention of rural physicians.

Acknowledgements We thank all the staff of the Division of Science and Education of Health Commission of Guangxi Province and all the faculty of the Division of Enrollment and Employment of GXMU who kindly helped collect the baseline data of both types of graduates and follow-up data of the RTME-GXMU graduates. We also thank Professor Lan lan for her important revisions on the language of this paper.

Contributors YS and YZ were the principal investigators responsible for the survey, and had full access to all the data in the study and took responsibility for the integrity of the data and the accuracy of the data analysis. Most authors (YS, XH, HL, EC, YK, JY, XL, YZ) contributed to the study conception, design, baseline investigation, follow-up survey and data collection. YS, EC and SAM performed the statistical analysis. YS wrote the manuscript. SAM helped revise the language. All authors discussed the findings and approved the final version for publication.

Funding This study was funded by the National Natural Science Foundation of China (grant number 71864006), the Natural Science Foundation of Guangxi Province (grant numbers 2020GXNSFAA238019 and 2018GXNSFAA138102) and the Higher Education Undergraduate Level Reform Program of Guangxi Province (grant number 2019JGA139).

Competing interests None declared.

Patient consent for publication Not required.

Ethics approval This study was approved by the Guangxi Medical University Ethics Commission and the written informed consent of each participant included in the study was obtained before any data were collected.

Provenance and peer review Not commissioned; externally peer reviewed.

Data availability statement All data relevant to the study are included in the article or uploaded as supplemental information.

Open access This is an open access article distributed in accordance with the Creative Commons Attribution Non Commercial (CC BY-NC 4.0) license, which permits others to distribute, remix, adapt, build upon this work non-commercially, and license their derivative works on different terms, provided the original work is properly cited, appropriate credit is given, any changes made indicated, and the use is non-commercial. See: http://creativecommons.org/licenses/by-nc/4.0/.
ORCID iDs

Ying Shen http://orcid.org/0000-0002-2434-5746

Xiaoyun Liu http://orcid.org/0000-0002-5483-0742

\section{REFERENCES}

1 Gu D, Zhang Z, Zeng Y. Access to healthcare services makes a difference in healthy longevity among older Chinese adults. Soc Sci Med 2009;68:210-9.

2 Long S. Primary care health professionals in rural China: current status, influential factors, and countermeasures. J Med Theory Pract 2013;7:878-9.

$3 \mathrm{Hou}$ J, Xu M, Kolars JC, et al. Career preferences of graduating medical students in China: a nationwide cross-sectional study. BMC Med Educ 2016;16:136.

4 National Bureau of Statistics of China. China statistical yearbook 2019. Beijing: China Statistics Press, 2019. http://www.stats.gov.cn/ tjsj/ndsj/2019/indexch.htm

5 National Health Commission of the People's Republic of China. China health statistical yearbook 2019. Beijing: Peking Union Medical College Press, 2019.

6 National Bureau of Statistics of China. China statistical yearbook 2012. Beijing: China Statistics Press, 2012. http://www.stats.gov.cn/ tjsj/ndsj/2012/indexch.htm

7 Rabinowitz HK, Diamond JJ, Markham FW, et al. Increasing the supply of rural family physicians: recent outcomes from Jefferson medical college's physician shortage area program (PSAP). Acad Med 2011;86:264-9.

8 Myhre DL, Bajaj S, Woloschuk W. Practice locations of longitudinal integrated clerkship graduates: a matched-cohort study. Can J Rural Med 2016;21:13-16.

9 Matsumoto M, Inoue K, Kajii E. A contract-based training system for rural physicians: follow-up of Jichi medical university graduates (1978-2006). J Rural Health 2008;24:360-8.

10 Jamar E, Newbury J, Mills D. Early career location of University of Adelaide rural cohort medical students. Rural Remote Health 2014;14:2592.

11 Nithiapinyasakul A, Arora R, Chamnan P. Impact of a 20-year collaborative approach to increasing the production of rural doctors in Thailand. Int J Med Educ 2016;7:414-6.

12 Huang X, Zuo YL, Tang Q. Studying on the implementation effect of rural-oriented medical education project in Guangxi. Chinese Healt Service Manag 2019;36:204-7.

13 Wendling AL, Phillips J, Short W, et al. Thirty years training rural physicians: outcomes from the Michigan state University College of human medicine rural physician program. Acad Med 2016;91:113-9.

14 Glasser M, Hunsaker M, Sweet K, et al. A comprehensive medical education program response to rural primary care needs. Acad Med 2008;83:952-61.

15 Sen Gupta T, Woolley T, Murray R, et al. Positive impacts on rural and regional workforce from the first seven cohorts of James Cook University medical graduates. Rural Remote Health 2014;14:2657.

16 Kwan MMS, Kondalsamy-Chennakesavan S, Ranmuthugala G, et al. The rural pipeline to longer-term rural practice: general practitioners and specialists. PLoS One 2017;12:e0180394.

17 Matsumoto M, Takeuchi K, Tanaka J, et al. Follow-Up study of the regional quota system of Japanese medical schools and Prefecture scholarship programmes: a study protocol. BMJ Open 2016;6:e011165.

18 Yi Y, Chongsuvivatwong V, Sriplung $H$, et al. CPIRD: a successful Thai programme to produce clinically competent medical graduates. F1000Res 2015;4:158.

19 Matsumoto M, Kajii E. Medical education program with obligatory rural service: analysis of factors associated with obligation compliance. Health Policy 2009;90:125-32.

20 Pagaiya N, Kongkam L, Sriratana S. Rural retention of doctors graduating from the rural medical education project to increase rural doctors in Thailand: a cohort study. Hum Resour Health 2015;13:10.

21 kataoka T, Takayashiki A, Sato M. Motives and factors associated with staying in medically underserved areas after the obligatory practice period among first-year Japanese medical students in a special quota system. Rural Remote Health 2015;15:3663 https:// jglobal.jst.go.jp/en/detail?JGLOBAL_ID=201902203876705645

22 Woolley T, Sen Gupta T, Bellei M. Predictors of remote practice location in the first seven cohorts of james cook university MBBS graduates. Rural Remote Health 2017;17:3992.

23 Quinn KJ, Kane KY, Stevermer JJ, et al. Influencing residency choice and practice location through a longitudinal rural pipeline program. Acad Med 2011;86:1397-406. 
24 Wheat JR, Leeper JD, Murphy S, et al. Educating physicians for rural America: validating successes and identifying remaining challenges with the rural medical scholars program. $J$ Rural Health 2018;34(Suppl 1):s65-74.

25 MacDowell M, Glasser M, Hunsaker M. A decade of rural physician workforce outcomes for the Rockford rural medical education (RMED) program, University of Illinois. Acad Med 2013;88:1941-7.

26 Crump WJ, Fricker RS, Ziegler C, et al. Rural track training based at a small regional campus: equivalency of training, residency choice, and practice location of graduates. Acad Med 2013;88:1122-8.
27 Wenghofer EF, Hogenbirk JC, Timony PE. Impact of the rural pipeline in medical education: practice locations of recently graduated family physicians in Ontario. Hum Resour Health 2017:15:16.

28 Playford DE, Evans SF, Atkinson DN, et al. Impact of the rural clinical school of Western Australia on work location of medical graduates. Med J Aust 2014;200:104-7.

29 Nicholson GC, Kondalsamy-Chennakesavan S. Determinants of rural practice: positive interaction between rural background and rural undergraduate training. Med J Aust 2015;202:477-9. 